\title{
Gastro-oesophageal reflux and dental erosion
}

\author{
What is the prevalence of dental erosion in patients who have \\ gastro-oesophageal reflux?
}

\author{
Pace F, Pallotta S, Tonini M, Vakil N, Bianchi Porro G. \\ Systematic review; gastroesophageal reflux disease and dental \\ lesions. Aliment Pharmacol Ther 2008; 27:1179-1186
}

Data sources Relevant studies were sourced using Medline and the Cochrane Controlled Trials Register, along with reference lists from identified papers and reviews.

Study selection Full papers were selected that assessed the prevalence of dental erosion in individuals who had gastro-oesophageal reflux (GERD), or vice versa, which were published in the English language.

Data extraction and synthesis Data extractions was carried out by at least two authors using a standardised data extraction form. Because of a marked variation in outcome measures, qualitative synthesis was carried out.

Results Seventeen studies met the selection criteria but they differed greatly in design, population, methods of diagnosing GERD, duration of followup and, consequently, in their findings. The median prevalence of dental erosion in GERD patients was $24 \%$, with a large range (5-48\%), and the median prevalence of GERD in dental erosion adults patients was $32.5 \%$ (range, $21-83 \%$ ) and $17 \%$ in paediatric patients (range, 14-87\%).

Conclusions This systematic review shows that there is a strong association between GERD and dental erosion. The severity of dental erosion seems to be correlated with the presence of GERD symptoms and also, at least in adults, with the severity of proximal oesophageal or oral exposure to an acidic $\mathrm{pH}$. The inspection of the oral cavity in search for dental erosions should become a routine manoeuvre in patients who have GERD.

\section{Commentary}

The loss of tooth surface may be through abrasion, attrition and/ or acid erosion. The "and/ or" indicates the multifactorial nature of tooth wear, also termed noncarious tooth surface/ substance loss (TSL). This multifactorial process presents a major difficulty when attempting to evaluate a single factor. Typically, an epidemiological index is used to assess extent and severity of erosion but the index cannot discriminate whether minimal loss of enamel or dentinal exposure is because of erosion, abrasion or attrition. The validity of measurements made using so-called erosion indices is questionable. The UK National Child Dental Health Survey of 2003 recognised this and dropped the term "erosion", used in the 1993 survey, in favour of TSL.

A small consensus group of interested parties devised, at a conference in 2007, a Basic Erosive Wear Examination index as a simple, reproducible scoring system and to assist in the decision-making process for the management of erosive tooth wear. Whether this index removes these difficulties remains to be seen, but the putative flaws of the indices may partly account for the generally weak associations between erosive factors and the outcome of erosion. The authors could not perform a meta-analysis because of the "marked variation in outcome measures" and stated that one study used the tooth wear index without discussion of its unsuitability for erosion measurement. Attempts to overcome index shortcomings have been to describe wear on labial or palatal surfaces as more likely to be from acid erosion, and to exclude incisal edge wear as this is likelier to be caused by attrition.

Of the 11 studies on adults included in the qualitative analysis, most had small sample sizes. Two study designs were assessed: prevalence of erosion in GERD cases, and prevalence of GERD in subjects with erosion. Diagnostic or "defining" criteria for GERD also varied, further adding to heterogeneity within the studies that were evaluated, a fact acknowledged by the authors.

The prevalence of erosion in adult subjects who had GERD ranged widely, from $5-48 \%$ in six studies (one study did not disclose the result): GERD ranged from $21-83 \%$ in the four studies assessing erosion cases. The results from the paediatric studies were even wider (13-87\%) and depended, according to the authors, on the method used to diagnose GERD. Reliability and comparability seem to be significantly compromised in the studies, although the review authors tended to play this down.

The authors conclude that the systematic review showed a strong association between GERD and erosion but the correlation coefficients cited were weak to moderate. The influence of confounding factors in the studies was not addressed, even though the authors mention that dietary acids are associated with erosion. It may be that GERD sufferers avoid acidic foodstuffs and therefore dietary acid is not an issue, but some mention of this would have been appropriate. There was no discussion of confidence in the results from the 17 studies that met their selection criteria.

In summary, the review could have been more critical of the studies. The association between GERD and dental erosion is entirely plausible but the strength of association and the epidemiological evidence remains unclear.

\section{Alex Milosevic \\ Liverpool University Dental Hospital, Liverpool, UK}

Evidence-Based Dentistry (2008) 9, 54. doi:10.1038/sj.ebd.6400586 